\title{
Neuronal Protein Alteration in Enteric Dysmotility Syndrome
}

\section{Irina Alafuzoff ${ }^{1,2 *}$, Svetlana N. Popova ${ }^{2}$, Alkwin Wanders ${ }^{3}$ and Be Ba Veress $^{4}$}

${ }^{1}$ Department of Immunology, Genetics and Pathology, Rudbeck Laboratory, Uppsala University, Uppsala, Sweden

${ }^{2}$ Department of Clinical Pathology, Uppsala University Hospital, Uppsala, Sweden

${ }^{3}$ Department of Clinical Pathology and Cytology, Umea University Hospital, Umea, Sweden

${ }^{4}$ Department of Clinical Pathology and Cytology, Skane University Hospital, Malmo, Sweden

\begin{abstract}
Little is known about the enteric ganglionic system in subjects with gastrointestinal dysmotility syndrome (GIDS). Furthermore, dysfunction of gastrointestinal motility is an early complaint of subjects with Parkinson's disease.

Here, we assessed p62/sequestosome-1(p62) and a-synuclein (aS) immunoreactivity (IR) in full-thickness bowel specimens of the gut obtained from six subjects with GIDS and from 17 controls.

In the myenteric neurons, fine punctuate p62-IR were seen in all of the controls, whereas diffuse cytoplasmic and nuclear p62-IR were seen in the GIDS cases. Physiological aS-IR (clone 42/aS) was seen in all of the controls and the GIDS cases in the lamina propria, the submucosal and in the myenteric plexuses. The disease associated aS (clone 5G4) labeled the cytoplasm of the ganglion cells only in the myenteric plexus in three out of the four subjects with the GIDS/inflammatory neuropathy.

In summary, ganglion cells were readily visualized in all of the layers of the bowel with clone 42/aS, and p62 displayed altered patterns of labeling in subjects with the GIDS. Labeling seen with the disease associated clone $5 G 4 / \alpha S$ in the GIDS/inflammatory neuropathy is intriguing and might indicate that the alteration of aS is triggered by a chronic inflammation.
\end{abstract}

Keywords: Gastrointestinal dysmotility; Immunohistochemistry; P62; Alpha-synuclein

\section{Introduction}

Gastrointestinal dysmotility syndrome (GIDS) can be the consequence of diseases involving the enteric nervous system (enteric neuropathies) or based on abnormalities of the enteric smooth muscles (myopathies) [1] Gastrointestinal (GI) functional disturbances are observed in subjects without any additional complaints but also in subjects with neurodegenerative diseases (ND) [2]. Studies carried out on subjects with Parkinson's disease (PD) have revealed that neuronal degeneration affects not only the neurons of the CNS but also the neurons located in the peripheral organs, i.e., disease associated a synuclein $(\alpha S)$ is seen in the visceral organs [3].

The p62/sequestosome-1(p62), an ubiquitin-binding scaffold/ adapter protein, has been shown to co-localize with the misfolded protein aggregates in the ND; thus, p62 has been recommended as a broad 'spectrum' detector of the inclusions while applying the immune histochemical (IHC) stain [4].

The objective of this study was to investigate whether p62 or $\alpha \mathrm{S}$ related ganglionic alterations are detected in the GIDS cases. We looked for p62 and aS immunoreactivity (IR) in the ganglionic cells in the controls and in subjects with GIDS. Regarding aS, two antibodies were used: directed towards the physiological and disease associated form.

\section{Materials and Methods}

Twenty-three full-thickness bowel specimens, from six patients with GIDS and 17 control subjects (normal bowel adjacent to the adenocarcinoma of the bowel that was the cause of the operation), were available for this study. The demographics of the subjects are summarized in Table 1. The preparation of the full-thickness bowel biopsies for diagnostics, measuring approximately $10 \times 15 \mathrm{~mm}$ on the serosal surface have been described in detail previously $[5,6]$.The typing of the six GIDS cases followed the recommendations by the Gastro
2009 Working Group and is primarily based on hematoxylin-eosin (HE) stained sections [1]. New $4 \mu \mathrm{m}$ thick sections were prepared from all of the cases for this study. None of the subjects had shown any signs or symptoms of a cerebral ND. The study was approved by the Uppsala Ethical Committee Dnr 2011/023 and 2011/289

\section{Immunohistochemistry}

For p62-IR, clone GP62-C was used (Progen Biotechnik GmbH, Heidelberg, Germany) at a dilution ratio of 1:100, pre-treatment autoclave $120^{\circ} \mathrm{C}$ in Ultra CCI Tris buffer, and the IHC staining was carried out in a BenchMark Ultra instrument (Ventana Medical Systems, Inc., Tucson, AZ). For detection, the peroxidase-conjugated streptavidin-biotin method was used (Dako Cytomation, Glostrup, Denmark) with DAB as chromogen. For physiological $\alpha$ S-IR, clone 42/ $\alpha \mathrm{S}$ (BD Transduction Laboratories, Franklin Lakes, NJ) and for disease associated aS clone 5G4/aS (Analytic Jena AG, Jena, Germany) were used at a dilution ratio of $1: 1000$, pre-treatment autoclave $120^{\circ} \mathrm{C}$ in citrate buffer, pH 6.0 followed by $5 \mathrm{~min}$ in $80 \%$ formic acid, and the IHC staining was carried out manually. For detection, Histostain ${ }^{\oplus}$-Plus $3^{\text {rd }}$ generation IHC detection Kit (Invitrogen, Carlsbad, CA) was used with Romulin AEC chromogen (Biocare Medical, Concord, CA).

*Corresponding author: Irina Alafuzoff, Department of Immunology, Genetics and Pathology, Uppsala University Hospital, Rudbeck Laboratory, Dag Hammarskjölds väg 20, 751 85, Uppsala, Sweden. Tel: +46 (0) 706114822; E-mail: irina.alafuzoff@igp.uu.se

Received January 08, 2016; Accepted February 10, 2016; Published February 16,2016

Citation: Alafuzoff I, Popova SN, Wanders A, Veress B (2016) Neuronal Protein Alteration in Enteric Dysmotility Syndrome. J Alzheimers Dis Parkinsonism 6: 212 doi: 10.4172/2161-0460.1000212

Copyright: @ 2016 Alafuzoff I, et al. This is an open-access article distributed unde the terms of the Creative Commons Attribution License, which permits unrestricted use, distribution, and reproduction in any medium, provided the original author and source are credited. 
Citation: Alafuzoff I, Popova SN, Wanders A, Veress B (2016) Neuronal Protein Alteration in Enteric Dysmotility Syndrome. J Alzheimers Dis Parkinsonism 6: 212. doi: 10.4172/2161-0460.1000212

\begin{tabular}{|l|l|l|c|c|}
\hline Case \# & with GIDS + I & Diagnosis & Gender & Age years \\
\hline 1 & + & Combined myoneuropathy & F & 44 \\
\hline 2 & + & Degenerative neuropathy & M & 46 \\
\hline 3 & + & Inflammatory neuropathy & F & 60 \\
\hline 4 & + & Inflammatory neuropathy & F & 56 \\
\hline 5 & + & Inflammatory neuropathy & F & 25 \\
\hline 6 & + & Inflammatory neuropathy & M & 31 \\
\hline 7 & - & Adenocarcinoma & M & 65 \\
\hline 8 & - & Adenocarcinoma & M & 55 \\
\hline 9 & - & Adenocarcinoma & M & 64 \\
\hline 10 & - & Adenocarcinoma & M & 57 \\
\hline 11 & - & Adenocarcinoma & M & 64 \\
\hline 12 & - & Adenocarcinoma & M & 69 \\
\hline 13 & - & Adenocarcinoma & M & 65 \\
\hline 14 & - & Adenocarcinoma & F & 69 \\
\hline 15 & - & Adenocarcinoma & M & 65 \\
\hline 16 & - & Adenocarcinoma & F & 66 \\
\hline 17 & - & Adenocarcinoma & F & 66 \\
\hline 18 & - & Adenocarcinoma & M & 54 \\
\hline 19 & - & Adenocarcinoma & F & 68 \\
\hline 20 & - & Adenocarcinoma & M & 67 \\
\hline 21 & - & Adenocarcinoma & M & 68 \\
\hline 22 & - & Adenocarcinoma & F & 53 \\
\hline 23 & - & Adenocarcinoma & F & 66 \\
\hline
\end{tabular}

GIDS: Gastrointestinal dysmotility syndrome; F: Female; M: Male

Table 1: Demographics of included subjects.

\section{Assessment}

All stained sections were assessed in light microscopy, and IR was registered as seen or not seen in magnifications ranging from X100 to X400. Ganglion cells located in the submucosal and myenteric plexuses were searched for in both HE and IHC stains. The pattern of IHC staining (granular cytoplasmic, diffuse cytoplasmic and nuclear) was assessed and representative photomicrographs were taken using an Olympus BX46 microscope and an Olympus UC30 camera. Images were acquired using the cellSens Entry software (Olympus Optical, Tokyo, Japan).

\section{Results}

The dichotomized outcome of the IHC stains is summarized in the Table 2.

In all 17 control subjects, finely punctuate cytoplasmic p62-IR (Figure 1A) was observed in all of the observed myenteric ganglion cells and granular labeling was seen in some of the cells in the lamina propria, but no labeling was observed in the submucosal ganglion cells. p62-IR was also observed in the myenteric ganglion cells in the subjects with GIDS as diffuse cytoplasmic labeling with or without small grains (Figure 1B and 1C), and as diffuse intranuclear p62-IR (Figure 1D).

Physiological 42/aS-IR was observed in the ganglion cells in all plexuses, in both controls and subjects with GIDS. In the controls, mainly diffuse cytoplasmic labeling with small grains was seen in the

\begin{tabular}{|c|c|c|c|c|c|c|}
\hline Type of gastrointestinal disease & $\mathbf{n}$ & Age range, years & Gender M/F & with p62-IR & with 42/aS-IR & with $5 G 4 / \alpha S-I R$ \\
\hline Inflammatory neuropathy & 4 & $25-60$ & $1 / 3$ & 4 & 4 & 3 \\
\hline Degenerative neuropathy & 1 & 46 & $1 / 0$ & 1 & 1 & 0 \\
\hline Combined myoneuropathy & 1 & 44 & $0 / 1$ & 1 & 1 & 0 \\
\hline With adenocarcinoma & 17 & $53-69$ & $10 / 7$ & $17^{*}$ & 17 & 0 \\
\hline
\end{tabular}

$\mathrm{N}$ : Number; M: Male; F: Female; IR: Immunoreactivity; * Only fine punctuate grains

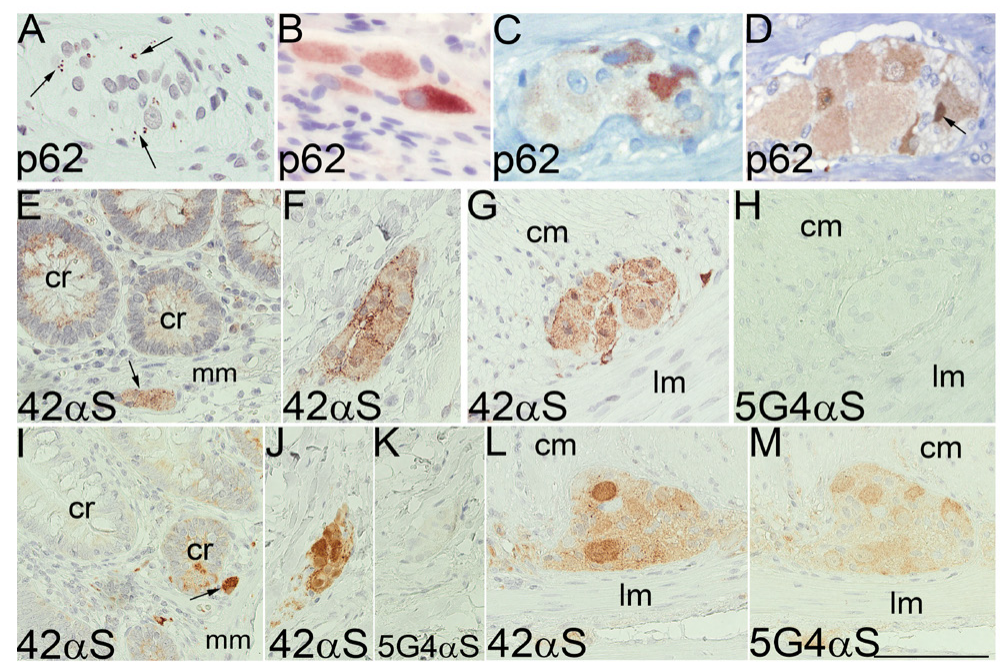

Figure 1: Immunohistochemical labeling of the enteric neurons

(A) p62- immunoreactivity (IR) seen as a minute granule in a control case (arrows). Note the myenteric ganglion cells showing diffuse cytoplasmic p62- IR in a case with inflammatory neuropathy (B) and in a case with degenerative neuropathy (C). Note, in addition to the cytoplasmic p62-IR, the labeling of the nucleus (arrow on D) in a case with degenerative neuropathy. 42/aS-IR ganglion cells of the mucosal plexus in close proximity to the muscularis mucosae (mm) in a control case (arrow on $\mathrm{E}$ ) and in a case with inflammatory neuropathy (arrow on I); submucosal ganglion in a control $(\mathrm{F})$ and in a case with inflammatory neuropathy (J). Note the absence of labeling in the submucosa in the case with inflammatory neuropathy when applying 5G4/aS (K). Extensive clone 42/aS-IR in the ganglion cells of the myenteric plexus in a control (G) and in a case with inflammatory neuropathy (L). Note 5G4/aS-IR in the cytoplasm in the case with inflammatory neuropathy (M), a labeling that was not observed in the control case $(\mathrm{H})$. cr - crypt; $\mathrm{cm}$ - circular muscle layer; Im - longitudinal muscle layer. Bar corresponds to $100 \mu \mathrm{m}$. 
cells in the lamina propria, whereas in the GIDS cases cytoplasmic labeling was somewhat stronger, suggesting an inclusion (Figure 1E and 1I). In the ganglion cells of the sub mucosal plexus, cytoplasmic granules were noted in the control cases when compared with more diffuse labeling of the cytoplasm in the GIDS cases (Figure $1 \mathrm{~F}$ and $1 J)$. A strong labeling with physiological $42 / \alpha S$ was detected in the numerous myenteric ganglion cells in both the controls and the GIDS cases (Figure $1 \mathrm{G}$ and $1 \mathrm{~L}$ ).

When applying the disease associated clone 5G4/aS, a faint cytoplasmic labeling was observed in three out of the six GIDS cases, all three being of the inflammatory neuropathy type (Figure 1M). This IR was observed only in the myenteric plexus, not in the lamina propria or the submucosa. None of the 17 controls displayed any IR applying the disease associated antibody (Figure $1 \mathrm{H})$.

\section{Discussion}

We assessed whether the alterations in the enteric neuronal system in the GIDS patient could be visualized using an antibody directed to the p62/sequestosome- 1 and compared the outcome with the controls. This approach was chosen since p62 has been recommended for use when screening for the eventual cellular aggregates of the altered proteins in ND [4]. Interestingly, p62-IR was seen in the controls as well as in the GIDS. The pattern of p62-IR, however, was somewhat differing. In the controls, only small granular cytoplasmic labeling was observed when compared with more diffuse and even nuclear labeling in the GIDS cases.

The observations described herein are similar to those described in the neurons in oxidative stress [7]. The accumulation of p62 has been suggested to serve as a marker for either the inhibition of autophagy, defects in the autophagic degradation, or autophagy induction [8]. Diffuse homogeneous cytoplasmic p62-IR has been suggested to represent the metabolizing misfolded proteins [9]. The diffuse cytoplasmic p62-IR might also indicate that the ganglion cells were exposed to damage (oxidative stress or damaging toxins from the lymphocytes) trying to overcome/repair the damage.

The expression of p62-IR in the nuclei of the myenteric neurons in some of our patients might suggest an abnormal accumulation of proteins within the nucleus. Noteworthy, nucleocytoplasmic shuttling of p62 has been demonstrated by Pankiv and colleagues [10], and intranuclear p62 aggregates have been reported to be seen in some types of ND [11]. Based upon our observations, we can conclude that p62-IHC indeed allowed for the visualization of an abnormal IR pattern in the ganglion cells in the GIDS cases assessed here.

It has been reported that in PD, $\alpha$ S-IR is seen not only in the brain but also in the visceral organs; moreover, in 2014, Gray and colleagues reported that $\alpha \mathrm{S} I R$ was also seen in the neurologically intact subjects $[3,12]$.

In line with previous reports, we observed a substantial clone $42 /$ aS-IR (physiological) in the ganglion cells both in the controls as well as in the GIDS cases. Physiological 42/aS-IR was observed in the lamina propria, the submucosa, and particularly in the numerous ganglion cells of the myenteric plexus. Our observations are in line with Gray and colleagues, indicating that all neurologically intact subjects display aSIR in their ganglion cell population [12]. Previous reports have reported aS-IR in none or in $8 \%$ to $82 \%$ of the neurologically intact subjects [11-15]. This discrepancy is related to the different antibodies used, different material assessed, i.e., surgical vs. post-mortem samples and the type of the sample, i.e., full thickness surgical sample vs. superficial endoscopic biopsy. Regarding antibodies, Gray and colleagues used a $\alpha \mathrm{S}$ clone MJFR1 (antigen aa 1-150) as opposed to the clone 42/aS antibody used here (antigen aa 15-123) [12]. Both of these antibodies cover a broad sequence of the $\alpha \mathrm{S}$ protein. Shannon and colleagues reporting an incidence of $8 \%$ used an antibody directed to the C-terminus of aS [14]. The significance of the antibody used was also recently emphasized in the work by Vaikath and colleagues [16]. It is worth noting that most studies reporting low incidence (0-8\%) of $\alpha S$-IR in the GI tract, in line with the work of Shannon and colleagues, have been carried out on superficial endoscopic biopsies lacking myenteric plexus $[13,14]$. Contrary, when whole thickness samples of GI obtained postmortem (containing myenteric plexus) have been assessed the incidence of $\alpha \mathrm{S}$-IR was reported being as high as $52 \%$ [15]. This emphasizes the significance of the type of material assessed, full thickness surgical sample vs. superficial endoscopic biopsy, while looking for the incidence of aSIR in GI tract. The difference in incidence of $\alpha S-I R$ in GI tract while assessing surgical samples (100\%) when compared to the postmortem samples (52\%) is most likely dependent on the fixation time. Previous reports have indicated that the postmortem time does not influence the aS-IR while applying IHC techniques, whereas a significant alteration of the IR has been reported due to the fixation time $[17,18]$. In routine setting the fixation time when handling surgical samples is relatively short (days) when compared with postmortem sampled tissue (weeks).

An interesting observation was the faint labeling of the ganglion cells in three out of the six GIDS cases when applying the disease associated clone 5G4/ $\alpha$ S. This "faint" 5G4/ $\alpha$ S-IR was observed in three out of the four GIDS cases with inflammatory neuropathy but not in the cases with degenerative neuropathy or combined myoneuropathy. This observation is unique and suggests that the alterations in the $\alpha \mathrm{S}$ might be related to a chronic inflammatory type of GIDS. This observation certainly merits further investigation of eventual aS alterations, particularly in subjects with chronic inflammatory bowel disorders.

The limitation of the current study is certainly the small cohort size of GIDS patients. One of the reasons to the limited number of cases is that subjects with dysmotility syndrome are seldom operated (in Sweden once per month in a population of 9 million subjects) in principle only when the symptoms are severe. Moreover to our knowledge no systematic assessment of the incidence/prevalence of GIDS has been carried out so far. Furthermore, complaints of a malfunction of the gastric motility are probably easily disregarded if not severe. Interestingly, the esophageal and GI motor dysfunction is reported often being present in PD and frequently precedes the onset of motor symptoms by more than one decade [19]. Whether these GI symptoms in PD are due to aS pathology is unclear, but noteworthy PD patients have been reported to display aSIR lesions in their GI tract [3].

Our results showed, that in GIDS of the inflammatory type, alteration/aggregation of aS was observed in the enteric neuronal system. This observation suggests that inflammation might trigger alteration/aggregation of $\alpha \mathrm{S}$. In order to test this presumption, IR of both the physiological and disease associated aS should be assessed in patients with various chronic inflammatory bowel disorders. Noteworthy, use of surgical samples and full thickness bowel biopsies seems to be as crusial as the choice of antibody while assessing aSIR in the GI tract $[10,13]$. Expression of both p62 and $\alpha$, should not only be assessed in the chronic inflammatory bowel disorders as suggested above, but also in GIDS cases as the results might shed light to the question raised, whether GIDS represent a subtype of aS-pathy. Furthermore, the use of conformation-specific aS antibodies might contribute to the unraveling of the development of aS pathology seen in the myenteric ganglion cells. 
Citation: Alafuzoff I, Popova SN, Wanders A, Veress B (2016) Neuronal Protein Alteration in Enteric Dysmotility Syndrome. J Alzheimers Dis Parkinsonism 6: 212. doi: 10.4172/2161-0460.1000212

\section{Acknowledgments}

We thank the medical laboratory technologists Maria Nilsson and Ivo Filinic at Skåne University Hospital for their skillful technical assistance and Meena Strömqvist for her critical reading of the manuscript. The study was approved by the Uppsala Ethical Committee Dnr 2011/023 and 2011/289. This study has been supported by the local ALF grants. The authors report no conflicts of interest.

Those responsible for the study design, assessment of the $\mathrm{IHC}$ and preparation of the manuscript - IA and VB; manual staining, assessment of the IHC and preparation of the manuscript - SP; and selection of the control cases - AW.

\section{References}

1. Knowles CH, De Giorgio R, Kapur RP, Bruder E, Farrugia G, et al. (2009) Gastrointestinal neuromuscular pathology: Guidelines for histologica techniques and reporting on behalf of the gastro 2009 international working group. Acta Neuropathol 118: 271-301.

2. Natale G, Pasquali L, Paparelli A, Fornai F (2011) Parallel manifestations of neuropathologies in the enteric and central nervous systems. Neurogastroenterol Motil 23: 1056-1065.

3. Beach TG, Adler CH, Sue LI, Vedders L, Lue L, et al. (2010) Multi-organ distribution of phosphorylated alpha-synuclein histopathology in subjects with Lewy body disorders. Acta Neuropathol 119: 689-702.

4. Salminen A, Kaarniranta K, Haapasalo A, Hiltunen M, Soininen H, et al. (2012) Emerging role of p62/sequestosome-1 in the pathogenesis of Alzheimer's disease. Prog Neurobiol 96: 87-95.

5. Lindberg G, Törnblom H, Iwarzon M, Nyberg B, Martin JE, et al. (2009) Fullthickness biopsy findings in chronic intestinal pseudo-obstruction and enteric dysmotility. Gut 58: 1084-1090.

6. Veress B, Nyberg B, Törnblom H, Lindberg G (2009) Intestinal lymphocytic epithelioganglionitis: a unique combination of inflammation in bowel dysmotility: a histopathological and immunohistochemical analysis of 28 cases. Histopathology 54: 539-549.

7. Selkoe DJ (2003) Folding proteins in fatal ways. Nature 426: 900-904.

8. Mariño G, Ugalde AP, Salvador-Montoliu N, Varela I, Quirós PM, et al. (2008) Premature aging in mice activates a systemic metabolic response involving autophagy induction. Hum Mol Genet 17: 2196-2211.
9. Braak H, Thal DR, Del Tredici K (2011) Nerve cells immunoreactive for $p 62$ in select hypothalamic and brainstem nuclei of controls and Parkinson's disease cases. J Neural Transm (Vienna) 118: 809-819.

10. Pankiv S, Lamark T, Bruun JA, Øvervatn A, Bjørkøy G, et al. (2010) Nucleocytoplasmic shuttling of p62/SQSTM1 and its role in recruitment of nuclear polyubiquitinated proteins to promyelocytic leukemia bodies. J Biol Chem 285: 5941-5953.

11. Pikkarainen M, Hartikainen P, Alafuzoff I (2008) Neuropathologic features of frontotemporal lobar degeneration with ubiquitin-positive inclusions visualized with ubiquitin-binding protein p62 immunohistochemistry. J Neuropathol Exp Neurol 67: 280-298.

12. Gray MT, Munoz DG, Gray DA, Schlossmacher MG, Woulfe JM (2014) Alphasynuclein in the appendiceal mucosa of neurologically intact subjects. Mov Disord 29: 991-998.

13. Ruffmann C, Parkkinen L (2016) Gut Feelings About Ît-Synuclein in Gastrointestinal Biopsies: Biomarker in the Making? Mov Disord 31: 193-202.

14. Shannon KM, Keshavarzian A, Mutlu E, Dodiya HB, Daian D, et al. (2012) Alpha-synuclein in colonic submucosa in early untreated Parkinson's disease. Mov Disord 27: 709-715.

15. Gold A, Turkalp ZT, Munoz DG (2013) Enteric alpha-synuclein expression is increased in Parkinson's disease but not Alzheimer's disease. Mov Disord 28 237-240.

16. Vaikath NN, Majbour NK, Paleologou KE, Ardah MT, van Dam E, et al. (2015) Generation and characterization of novel conformation-specific monoclonal antibodies for Ît-synuclein pathology. Neurobiol Dis 79: 81-99.

17. Alafuzoff I, Parkkinen L, Al-Sarraj S, Arzberger T, Bell J, et al. (2008) Assessment of alpha-synuclein pathology: a study of the BrainNet Europe Consortium. J Neuropathol Exp Neurol 67: 125-143.

18. Pikkarainen M, Martikainen P, Alafuzoff I (2010) The effect of prolonged fixation time on immunohistochemical staining of common neurodegenerative disease markers. J Neuropathol Exp Neurol 69: 40-52.

19. Pellegrini C, Antonioli L, Colucci R, Ballabeni V, Barocelli E, et al. (2015) Gastric motor dysfunctions in Parkinson's disease: Current pre-clinical evidence. Parkinsonism Relat Disord 21: 1407-1414.
Citation: Alafuzoff I, Popova SN, Wanders A, Veress B (2016) Neuronal Protein Alteration in Enteric Dysmotility Syndrome. J Alzheimers Dis Parkinsonism 6 : 212. doi: $10.4172 / 2161-0460.1000212$
OMICS International: Publication Benefits \& Features

Unique features:

- Increased global visibility of articles through worldwide distribution and indexing

- Showcasing recent research output in a timely and updated manner

Special issues on the current trends of scientific research

Special features:

700 Open Access Journals

50,000 editorial team

Rapid review process

Quality and quick editorial, review and publication processing

Indexing at PubMed (partial), Scopus, EBSCO, Index Copernicus and Google Scholar etc

- Sharing Option: Social Networking Enabled

- Authors, Reviewers and Editors rewarded with online Scientific Credits

Better discount for your subsequent articles

Submit your manuscript at: http://www.omicsonline.org/submission/ 